\title{
LA EDUCACIÓN EN PARAGUAY EN TIEMPOS DE COVID-19
}

\author{
Paraguay education in COVID-19 times
}

\author{
Derlis Alfredo Noguera \\ Secretario general del Ministerio de Educación y Ciencias y miembro secretario ejecutivo \\ del Consejo Nacional de Educación Superior de Paraguay (Paraguay)
}

El coronavirus es una realidad que trajo consigo un replanteamiento para cada uno de los habitantes de la tierra. Los proyectos, el trabajo, la economía, nuestra agenda han quedado suspendidos o desaparecieron, esta crisis nos obliga a adoptar otro estilo de vida acorde con los cambios que enfrentamos. Con gran parte de la población mundial forzada a realizar teletrabajoy muchos que se han quedado sin empleo, racionalizamos más los recursos moderando nuestro consumo, obligados a trabajar y a vivir pensando en la salud y en el bienestar de todos los que nos rodean. El sistema educativo nacional debe responder a un cambio de paradigma en la enseñanza y el desarrollo de las clases, así como en la evaluación de conocimientos y competencias. Los órganos estatales encargados de establecer las políticas educativas deben desarrollar a corto plazo las medidas de contingencia necesarias para garantizar el bienestar de la comunidad educativa en general y a su vez asegurar el acceso a la educación, para lo cual las tecnologías de la información y comunicación se constituyen en un factor clave. Se necesita la colaboración de todos los sectores educativos, tal como lo indica nuestra carta magna: «La educación es responsabilidad de la sociedad y recae en particular en la familia, en el municipio y en el Estado». Nos encontramos con el desafío de desarrollar la resiliencia necesaria para entender los nuevos retos y adaptarnos a ellos.

Palabras clave

Educación, enseñanza, tecnología, resiliencia, responsabilidad
The Coronavirus is a reality that brought with it a rethinking for each of the inhabitants of the earth; projects, work, economy, our agenda has been suspended or disappeared, this crisis forces us to adopt another lifestyle, according to the changes we face. With a large part of the world's population forced to do telework and many who have become unemployed, we are further rationalizing resources by moderating our consumption, forced to work and live with the health and well-being of all those around us in mind. The national educational system must respond to a change of paradigm in teaching and class development, as well as in the evaluation of knowledge and skills; the state bodies responsible for establishing educational policies must develop in the short term the necessary contingency measures to guarantee the welfare of the educational community in general and in turn ensure access to education, for which Information and Communication Technologies are a key factor. Collaboration is needed from all educational sectors, as indicated in our Magna Carta: "Education is the responsibility of society and falls in particular on the family, the municipality and the state". We are faced with the challenge of developing the resilience necessary to understand and adapt to new challenges.

Keywords

Education, teaching, technology, resilience, responsibility 


\section{Introducción}

Resulta claro que en la actualidad se tienen muchos más recursos que en décadas anteriores, lo cual todo hablando desde el punto de vista educativo, la tecnología se constituye en una herramienta fundamental que posibilita el desarrollo de las clases a distancia y de forma virtual. La educación en línea distancia y de forbilidad como forma alternativa o complementaria a la modalidad presencial y no se puede negar el valor que tiene este instrumento hoy, cuando se deben afrontar situaciones críticas.

Es verdad también que existen varios cuestionamientos sobre si la educación online es la solución. Es probable que no sea la única, pero es una solución. Técnicamente, es mucho más posible de lo que algunos se imaginan. En España hay universidades que desarrollan la modalidad en línea y cuentan con un alto nivel cualitativo, entre las que podríamos destacar la UOC (Universidad Oberta de Catalunya) o la UNED (Universidad Nacional de Educación a Distancia), y universidades presenciales con capacidad para transformar la enseñanza presencial en virtual a través de plataformas y herramientas digitales. Sin embargo, el reto que se plantea no es solo hacerlo, sino hacerlo bien y de manera efectiva. Para que toda la oferta educativa tenga un alto nivel de calidad, son necesarias una capacitación y una experiencia notables.

A veces se entiende la educación online de forma muy simplista. Se piensa que con solo sustituir el medio (presencial) por otro tecnológico (plataforma, vídeo, etcétera) ya es suficiente. Eso demuestra un profundo desconocimiento de lo que significa la educación online de calidad. Quien piense que grabando las clases magistrales del profesorado ya tiene un curso online es que no sabe lo que es la verdadera educación online. La que resulta verdaderamente efectiva es la que se basa en la generación de interacciones y mediaciones entre estudiantes y profesores, el uso de recursos digitales de alta calidad pedagógica (desde vídeos a simulaciones, llegando hasta la realidad virtual) y el trabajo en equipo de docentes y estudiantes a partir a diseño educativo de cada curso con un alto grado de consistencia. No es una cuestión meramente tecnológica. La tecnología es el soporte, el modelo pedagógico es el valor clave.

Hay que innovar, pero no solo en términos de tecnología, con mayor razón en un mundo globalizado

una competitividad extrema. (Anelli, 2020).
Resulta necesario educar y formar a las generaciones cada vez más en torno a la posibilidad de nuevas sión por vivir der todo lo grande que ofrece el mundo a través del saber de la ciencia y de la verdad detrás de cada realidad. En este contexto, el trabajo de los profesores y docentes puede cobrar una dimensión mucho más auténtica, capaz de poner las preguntas justas de educar uniendo contenidos, habilidades, destrezas $y$ afectos alrededor de una curiosidad atenta crecer en el saber y en el saberse en esta socied trabajando con otros por la construcción de la casa común.

Paradójicamente, hace aproximadamente cien años, en el 1918, se vivió un acontecimiento similar con la llamada gripe española, cuya denominación se debió a que la mayor cantidad de información respecto a la enfermedad fue la proveniente de la peninsula ibérica, lo cual hizo suponer que había tenido su origen allí. Hasta hoy, resulta imposible determinar con exactitud el impacto de esa pandemia las estimaciones hablan de un número máximo de quinientos millones de personas afectadas y un numero de muertes no determinado, estimado entre cincuenta y cien millones de personas (Velázquez Seiferheld, 2020

En octubre de 1918, llegó la pandemia de la «gripe española» a Paraguay y la situación del país en materia de salud estaba muy lejos de ser óptima: según Milda Rivarola, solo existían 52 médicos: 50 en la capital y 2 en Villarrica; y cuatro quintas partes de la población vivia sin ningún tipo de atención médica profesional; es más, la Facultad de Medicina había sido clausurada por falta de alumnos (Velázquez Seiferheld, 2020)

En esas circunstancias, se adoptaron las primeras medidas estructurales: se resolvio que la Sanidad Fluvial proceda a la observación de barcos, el aislamiento de los enfermos y sospechosos, y la notificación las autoridades sanitarias. Los diarios se hicieron eco de las preocupaciones del departamento y divulgaron medidas de prevención y atención. Se recomendaba, por ejemplo, el blanqueo de las habitaciones a la cal y la desinfección de pisos con creolina. Se sugería purificar el agua a traves del hervido, filtración (usando filtro de porcelana porosa) o por el tratamiento químico del agua. De todos modos, el primer caso fatal ocurrio el 11 de diciembre de 1918.

Los lugares públicos de Asunción -como cinematógrafos, teatros y circos- fueron temporalmente in habilitados y desinfectados. En la Tablada, en el mercado central y en todos los puestos de venta, carne frutas y verduras de la capital se adoptaron medidas Especiales (Velázquez Seiferheld, 2020).

En cuanto al sistema educativo, se adelantaron los exámenes en todas las escuelas y colegios de la república. Simultáneamente, ocurría lo mismo en la Argentina (Velázquez Seiferheld, 2020).

Aun así, a pesar de las medidas adoptadas, cuando, en agosto de 1919, se declaró el final de la pandemia, habían fallecido -según datos oficiales516 personas en Asunción y más de 1.500 personas n el resto del país; en total, más de 2.000 personas, quizás entre el $0,2 \%$ y el $0,4 \%$ de la población tote, muy pocos niños y niñas fallecieron a causa dicha enfermedad. En la región el escenario era siguiente: en el Brasil, se estima que la tasa de mortandad llegó hasta el $2 \%$ en determinadas pobla ciones y produjo una crisis política de primer orden mientras que en la Argentina la mortalidad alcanzó mientras que en la Argentina la mortalidad alcanz a aproximadamente 13.000 personas, lo que repreguay las estimaciones rondan el $0.4 \%$ (Velázquez Seiferheld, 2020).

Volviendo a la realidad actual, debemos recordar que todas las medidas que se llevan adelante en este momento -sobre todo las educativas- son medidas de contingencia, es decir, provisorias para sobrellevar esta pandemia, para lo cual se necesita la colaboración de todos los sectores; es más, quiero recordar lo que indica nuestra carta magna en el artículo 75: «De la responsabilidad educativa. La educación es responsabilidad de la sociedad recae en particular en la familia, en el municipio en el Estado».

Francesco Tonucci, pedagogo experto en niños, nos dice que actualmente existe un conflicto entre escuela y familia; es un conflicto moderno, la familia siempre está lista para denunciar al colegio. Ahor la situación es nueva: la escuela se hace en familia, en casa. Propongo que la casa se considere un laboratorio donde descubrir cosas y los padres sean colaboradores de los maestros. Por ejemplo, como funciona una lavadora, tender la ropa, planchar aprender a coser.

Otro ejemplo con relación a la lectura, continúa Tonucci: hay otra forma, que es la lectura colectiva, de familia. Esta actividad puede ser propuesta por los mismos docentes. Crear un teatro que tiene su horario y su lugar en la casa, donde un miembro de la familia lee un libro como si fuera una telenovela. Media hora todos los dias. Son propuestas que parecen poco escolares, pero todas tienen quever con las disciplinas escolares. Estudiando la planta de la casa, se puede hacer una experiencia de geometría. Todo esto lo digo para que se entienda que se puede aprovechar la riqueza que tenemos ahora: la casa y la disponibilidad de los padres. Algunos dicen que los padres no tienen tiempo, pero no es verdad. A pesar de todo el tiempo que están ocupados, no saben qué hacer en el tiempo libre. Normalmente el tiempo que pasan con ellos es para acompañarlos a actividades y no para vivir con ellos. Otra propuesta es que jueguen, eso es lo más impre inventen juegos. Llamar a los abuelos para que aconsejen juegos, ellos fueron niños cuanlos juegos había que inventarlos (Tonucci, 2020). Otro dato importante de la realidad es aquello que tiene que ver con el punto de vista económico y financiero. Esta crisis trae consigo una merma en el ingreso de muchas familias, muchos empresarios se están viendo en la necesidad de cerrar sus empresas y por lo tanto muchos empleados están perdiendo sus trabajos. En esta situación crítica que afecta sobre todo al sector privado, ¿cómo se sostiene economicamente la educación privada si tenemos en cuenta que muchas familias se verán imposibilitadas de soportar la obligación de los pagos de las cuotas?

Todos debemos tomar conciencia sobre esta realidad económica que va a golpear fuerte a muchos, por eso es importante que -desde el punto de vista de las instituciones educativas privadas- la comunidad educativa en su conjunto-directivos, docentes, padres y estudiantes- pueda asumir un

Las instituciones educativas de gestión privada por su parte, deben verificar al interno de su administración y considerar -mirando todos los factores de la realidad- una reducción en los aranceles. Asi también quienes están obligados a abonar las cuotas tendrán que saber que se pide un esfuerzo muy grande para sostener la educación paraguaya. Las instituciones educativas privadas constituyen a lo largo de la historia un sosten fundamental para el sistema educativo nacional.

Desde el Gobierno nacional ya se están realizando reuniones con todos los actores del sector público y privado para verificar las distintas alternativas en relación al tema financiero, como becas, créditos y posibilidad de subsidios, entre otras.

Por eso es que esta situación de crisis nos obliga a buscar un consenso entre todos los actores de la educación $\mathrm{y}$, en todos los niveles, todos debemos ser protagonistas de este momento historico, en el cual la realidad nos pide buscar lo esencial de las cosas que vivimos.

Como nos recuerda el pensador y sacerdote español Julián Carrón, «nos hallamos ante un desafío sin precedentes para nuestra generación. La situación que estamos viviendo nos ha hecho conscientes de que en estos años hemos vivido en cierto sentido como en una burbuja que nos hacia percibirnos protegidos de los golpes de la vida. De este modo hemos vivido distraídos, fingiendo que todo estaba bajo control. Pero las circunstancias han desbaratado nuestros planesy nos han llamado bruscamente a responder, a tomarnos en serio nuestro yo 
a preguntarnos sobre nuestra situación existencial concreta» (Carrón, 2020).

\section{La educación en los niveles inicial, básica y media}

Si nos adentramos en las medidas adoptadas por el Gobierno nacional a través del Ministerio de Educación y Ciencias, nos encontramos que se determinó la suspensión de las clases por resolución num. 308/2020 con fecha 10 de marzo de 2020 en las instituciones de gestión oficial, privada y privada subvencionada, asi como el desarrollo de planes gias institucionales, a fin de evitar la expansión de gias institucionales, a fin de evitar la expansion de (COVID-19) para garantizar el bienestar de los estudiantes, personal docente y comunidad educativa en general; dicho período de suspensión de clases presenciales se fue extendiendo, de conformidad con las disposiciones de aislamiento, a través de las resoluciones núm. 354 con fecha 28 de marzo y 375 con fecha 12 de abril de 2020.

Es así que, dentro de las medidas de contingencia, el Ministerio de Educación y Ciencias (MEC) fue indicando los mecanismos a ser desarrollados para la continuidad de las actividades educativas y otras situaciones no menos importantes, como son: I) proveer, independientemente a la suspensión de las clases, kits de alimentos básicos, que se componen de desayuno, almuerzo y merienda, a aquellos alumnos que se encuentran dentro del programa de alimentación escolar, estableciendo -a través de resolución ministerial-como criterio de entrega de los citados kits la prioridad de las zonas vulnerables y de escasos recursos; II) la disposición de contar con todos los datos actualizados de los estudiantes a través de Registro Unico del Estudiante (RUE); III) la implementación de plataformas virtuales; entre otras.

Teniendo en cuenta que ya nos hemos referido al tema lineas arriba, vamos a empezar comentando lo referente a las plataformas virtuales habilitadas por el Ministerio de Educación y Ciencias para el desarrollo de las clases.

Desde el Ministerio de Educación y Ciencias fueron habilitadas dos plataformas virtuales para el desarrollo de las clases y la continuidad del calendario académico:

La herramienta Microsoft Teams de la plataforma Office 365, la cual es utilizada de manera obligatoria para trabajos colaborativos, comunicacion y videoconferencias entre las distintas unidades deI MEC central, sus instancias descentralizadas e instituciones educativas. Su utilización es exclusivamente para fines educativos, investigaciones o de apoyo a las actividades académicas. Esta platafor- ma permite una interacción muy interesante entre e docente y el estudiante, así como la carga de las clases de acuerdo con cada disciplina y con la posibilse presenta como una herramienta muy útil para el abordaje pedagógico.

La plataforma de recursos digitales denominada u Escuela en Casa, para estudiantes de los niveles inicial, escolar básica y media, como mo de apoyo para el desarrollo de las cladel sitio web huciones educativas del país, a través plataforma cuenta con distintas variables pedagógcas, desde una biblioteca de recursos virtuales a cual los estudiantes pueden acceder hasta sistemas de aprendizaje en distintas áreas, como matemáticas, y clases virtuales.

Es importante resaltar que las plataformas de Ministerio de Educación y Ciencias cuentan con red de acceso libre, lo cual es posible en el marco de un convenio con las empresas de telefonía proveedoras del servicio de Internet, que fue gestionado por el MEC; por lo tanto, es un instrumento de carácter público y gratuito. Así también, se implementó el Canal Educativo, que se trasmite por muchos canales de aire -canales que se están sumando esta iniciativa educativa en este tiempo de crisisen donde se desarrollan las clases diarias que se han alojado en la plataforma. Cabe destacar que las clases son presentadas por docentes con los contenidos educativos para los niveles inicial, básica y media.

Para el caso de las instituciones educativas que no son de gestión oficial, el MEC, como órgano rector del sistema educativo nacional, dispuso que las instituciones educativas de gestión privada y privada subvencionada podrán implementar y aplicar herramientas tecnológicas o digitales de enseñanza-aprendizaje para el desarrollo de los contenidos curriculares, a los efectos de asegurar el servicio educativo a los estudiantes y el cumplimiento de calendario academico correspondiente, como medida de contingencia en el marco de la emergencia sanitaria declarada ante el riesgo de expansión del coronavirus (COVID-19) en el territorio nacional.

Resulta claro que desde el Gobierno naciona se considera pertinente y oportuno recurrir a la implementación de las tecnologías de la información y comunicación como herramienta válida para dar continuidad al desarrollo de los contenidos curriculares correspondientes al presente año lectivo teniendo en cuenta que la suspensión de clases presenciales posiblemente llegue hasta el mes de diciembre de 2020, de manera a garantizar el bieestar de los estudiantes y la comunidad educativa en general.
Reconociendo las limitaciones que pueden preentarse en la utilización de las plataformas, desde punto de vista personal, considero que el Ministerio de Educación y Ciencias está ofreciendo las alternativas para cubrir a todos los sectores y evitar que lo estudiantes dejen de aprender las nociones académicas básicas en este período. Es así que, por ejemplo, aquellos alumnos de instituciones educativas que se encuentren en situación de vulnerabilidad quienes les resulte imposible acceder a la plataforma o medios tecnológicos podrán, en su institución educativa, retirar un material impreso con las clases y tareas a ser desarrolladas y acompañar el desarrollo de las clases en el canal educativo en cualquier canal de aire como mencionábamos anteriormente. Desde el inicio de la cuarentena en el Paraguay (10 de marzo de 2020), se implementaron medidas de contingencia y coordinación de las estrate gias a ser adoptadas para garantizar la prestación del servicio educativo, disponiendo la utilización de las tecnologías de información y comunicación y la distribución de materiales impresos como herramientas válidas para dar continuidad al año lec tivo. Las herramientas destinadas a evitar la pérdid del año lectivo se sustentan en la implementación coordinada de tres estrategias digitales. Dichas es trategias digitales se definen como de acceso: las cuales permiten a los alumnos y docentes la descarga de materiales y el acceso a recursos educativos; de interacción: donde los mismos participan activamente en aulas virtuales; $y$, finalmente, las de difusión: que incorporan los medios masivos de comunicación, como la televisión, la radio y otros; y en forma excepcional, para aquellos casos donde existan impedimentos para el acceso a medios digitales, se aplica una cuarta estrategia, que incluye la entrega física de los materiales a los estudiantes a fin de consolidar el proceso de enseñanza-aprendizaje distancia, amparado en lo dispuesto en la Ley Núm. 1264/1998 General de Educación, la cual establece la educación a distancia en sus artículos 59 y 60, res pectivamente.

En un contexto de emergencia sanitaria, las cuarentenas sostenidas o intermitentes motivan la toma de decisiones que involucran, principalmente, el viraje de la tradicional y casi única conocida modaidad presencial a una modalidad de educación distancia o virtual que incluye la distribución de materiales en formato fisico, además de una inmediata aplicación de estrategias para la disponibilidad de materiales y recursos, repositorios de contenidos digitales, la formación de los docentes para el acceso, afianzamiento en el uso y generación de conocimientos mediante las plataformas educativas, las adaptaciones de desarrollo curricular, la atención a la disponibilidad de disposivivos con los que se llega a la formación de los estudiantes.
Desde el Gobierno nacional se considera pertinente y

oportuno recurrir a la implementación de las

tecnologías de la información

y comunicación como

herramienta válida para dar

continuidad al desarrollo

del presente año lectivo

Teniendo en cuenta todos los factores mencionados precedentemente, es que el MEC consideró oportuno disponer un receso en las actividades academicas por medios virtuales desde el 4 hasta ción Núm. 430 con fecha 30 de abril de 2020, a los efectos de continuar estableciendo y mejorando las estrategias académicas y evaluar los mecanismos ya implementados a través de las modalidades a distancia o virtual en las instituciones educativas de gestión oficial de los niveles y modalidades correspondientes al viceministerio de Educación Básica.

Otra de las acciones llevadas adelante por el Ministerio de Educación y Ciencias como medida de contingencia ante la suspensión de las clases presenciáles-acción determinada ante el riesgo de expansión de la enfermedad COVID-19- es la que los cou ver con la alimentación escolar, ya que en los colegios oficiales los estudiantes, sobre todo aquellos de escasos recursos, reciben suplementos muerzo y merienda.

De conformidad con la Ley Núm. 5210, «De alimentación escolar y control sanitario", se dispone que la autoridad de aplicación de la presente ley es el Ministerio de Educación y Ciencias, que coordinará las acciones pertinentes con las demás instituciones nacionales o locales involucradas.

Acorde con las facultades otorgadas por la normativa citada precedentemente, el Ministerio de Educacion y Ciencias consideró oportuno continuar ofreciendo la alimentación escolar a los estudiantes y para ello estableció la composición de kits de alimentos para entregar a cada estudiante. También elaboro un protocolo para la entrega de los kits de alimentos de manera excepcional por el período de tiempo que duren las medidas sanitarias. 


\section{Educación superior}

El Instituto Internacional de la Unesco para la Educación Superior en América Latina y el Caribe (IESALC) ha preparado un informe sobre los impactos del COVID-19 en la educación superior, el cual refleja explícitamente la situación en la cual nos encontramos como región para afrontar esta crisis so-

Así, este informe nos proporciona primeramenun dato curioso: indica que, desde su fundación las universidades, como que, descer sundación social, han tenido que enfrentarse a epidemias devastadoras que han impactado en su funcionamiento cotidiano. Y han sobrevivido y continuado con su misión aun con las puertas cerradas. En 1665 la Universidad de Cambridge cerró por causa de una epidemia de peste negra que azotó Inglaterra. Isaac Newton tuvo que volver a Woolsthorpe Manor, su hogar. Un día, sentado en el jardín, vio caer una manzana que le inspiró para formular su teoría de la gravitación universal o, por lo menos, así se lo contó a William Stukeley, quien incluyó esta anécdota en la biografía de Newton que publicaría tras su muerte. La moraleja de esta historia es que, por mucho que haya que cerrar las puertas de las instituciones de educación superior, las actividades académicas continúan allí donde hay espíritus comprometidos con la ciencia y la formación y, a veces, con sorprendentes resultados. Por cierto, la Universidad de Cambridge ha vuelto a cerrar sus puertas ahora en 2020, por segunda vez en su historia (Unesco IESALC, 2020).

Actualmente, los cierres temporales de instituciones de educación superior (IES) por causa de la pandemia del COVID-19 ya dejaron de ser noticia, porque ya son mayoria los paises donde han dejado de operar presencialmente. Las estimaciones de Unesco IESALC muestran que el cierre temporal afecta aproximadamente a unos 23,4 millones de estudiantes de educación superior (CINE 5, 6, 7 y 8 ) y a 1,4 millones de docentes en America Latina y el Caribe; esto representa, aproximadamente, más del $98 \%$ de la población de estudiantes y profesores de educación superior de la región.

En America Latina -siempre conforme al análisis emitido por el Instituto Internacional para la Educación Superior de America Latina y el Caribe (IESALC) en cuanto a la Educación Superior- las medidas de confinamiento o cuarentena se tomaron practicamente de forma inmediata y, en algunos casos, con una perspectiva temporal larga (Unesco IESALC, 2020).

Así, por ejemplo, en Argentina se recomendó la suspensión de las clases presenciales el 14 de marzo; en Chile, la cuarentena total en algunas comu-
nas se ha traducido en un cierre masivo de las IES a partir del 16 de marzo; en Colombia, la totalidad de las IES están cerradas siguiendo el decreto de emergencia sanitaria del 12 de marzo, previsiblemente hasta el 30 de mayo: en Cuba se clausuraron las IES el 25 de marzo por un período indeterminado; en Perú se suspendieron las clases presenciales el 12 de marzo, en un primer momento solo por quince días, pero actualmente se ha ampliado la suspensión de las clases presenciales hasta 4 de mayo; en El Salvador el 11 de marzo se suspendieron las clases por treinta días hasta nuevo aviso: en Uruguay, la Universidad de la República ordenó el cese de actividades presenciales el 15 de marzo también por casi treinta días: en Venezuela estado de alarma se decretó ya el 13 de marzo, inicialmente por treinta días. A día de hoy, en la región solo algunas IES en Brasil y México parecen seguir abiertas (Unesco IESALC, 2020).

Nadie sabe a ciencia cierta cuánto tiempo pueden durar estos cierres. Las medidas iniciales tomadas por muchos gobiernos han oscilado entre quince y treinta días, pero es fácil anticipar que esta duración se extenderá hasta que la pandemia remita. No es descabellado imaginar escenarios en los que esta situación se prolongue dos meses o más o como ya se ha anunciado en España e Italia, se decida no reanudar las clases presenciales en lo que queda de curso académico, que allí finaliza normamente en el mes de junio (Unesco IESALC, 2020).

En el mismo sentido, en Paraguay, al igual que en los niveles de educación inicial, básica y media, sin dudas, la educación superior ha visto afectadas sus actividades presenciales, donde, claramente con el objetivo sanitario de evitar la propagación del COVID-19, el Consejo Nacional de Educación Superior (CONES), institución rectora de la educación superior del Paraguay creada por la Ley Núm. 4995/2013, también tomó determinaciones importantes para este periodo de cuarentena provocado por el virus de COVID-19. La primera de ellas tiene que ver con la suspensión de clases presenciales, conforme a lo estipulado por el Gobierno nacional con igual ciclo que para los demás niveles educativos, es decir, desde el 11 de marzo de 2020.

Así también, el CONES ha dispuesto -resoluciones mediante- la posibilidad de que las instituciones de educación superior (IES) también puedan implementar mecanismos tecnológicos para el desarrollo de las clases y la continuidad del calendario académico.

Por lo tanto, se dispuso que las instituciones de educación superior, sujetas a la Ley Núm. 4995/2013, podrán implementar y aplicar las herramientas digitales de enseñanza-aprendizaje en sustitución de las clases presenciales, a fin de desarrollar los contenidos de las asignaturas o disciplinas de las carreras de grado y programas de postgra- do -legalmente habilitados- siempre y cuando la naturaleza y condiciones de las mismas lo permitan y a adémico de las entidades educativas.

Teniendo en cuenta que -como en todos los nivtoridades son medidas de contingencia que se están desarrollando con el firme objetivo de responder a la realidad de crisis que se está viviendo en el mundo, también en este sentido en la educación superior se ha visualizado que muchos estudiantes manifiestan su descontento con el desarrollo de las clases a través de los medios tecnológicos y utilizan prácticamente los mismos válidos argumentos manifestados por los estudiantes de los demás niveles educativos: dificultad en el acceso a la conectividad, inexistencia de plataformas virtuales por parte de las IES, poca capacitación de los docentes para el desarrollo de las clases a través de esta modalidad y sobre todo, y quizás la más preocupante, dificultad del desarrollo de las prácticas para aquellas materias que lo requieren, como las carreras del área de ciencias de la salud e ingeniería y sus combinaciones.

La situación actual ha motivado a varios estamentos de la educación superior a generar encuentros virtuales para buscar opciones eficaces y aplicables en la premura existente con las que responder a los reclamos y circunstancias que se están suscitando en la realidad académica de la educación superior; estos encuentros están liderados por el Ministerio de Educación y Ciencias a través de su representación en el Consejo Nacional de Educación Superior (CONES) y del viceministerio de Educación Superior y Clencias, conjuntamente con representantes das, de institutos de educación superior público y privados, de rectores de universidades públicas y privadas, como tambien del Consejo de Rectores y de Directores Generales de Institutos de Educación Superior.

Estas reuniones han servido para que varios de los actores de la educación superior, que en su mayoría forman parte del ente rector de la educación superior que es el Consejo Nacional de Educación Superior (CONES), por supuesto respetando esa condición del CONES de ente rector, ya que es que tiene potestad de tomar las determinaciones, puedan tomar conciencia de cuál es el posible camino a seguir ante esta crisis, asumiendo todos juntos el compromiso de responder a esta realidad.

Entre las determinaciones asumidas en los citados encuentros, de las cuales se informó debidamente a la comunidad educativa, se pueden destacar:

a) La necesidad de planificar y realizar los ajuste en los calendarios académicos del 2020. b) La implementación de clases presenciales escalonadas -cuando se autorice la reanudación de tarios correspondientes para el ingreso a las aulas. c) El desarrollo de clases virtuales mediante c) El desarrollo de clases virtuales mediante plaInternet por parte los estudiantes.

d) La necesidad de instalar una mesa de diálogo con las empresas de telefonía para la liberación de Internet en el uso de las plataformas para el desarrollo de las clases, de tal forma que se atienda a los casi 300.000 estudiantes que forman parte del subsistema de educación superior.

e) La necesidad de capacitar a los docentes y a los alumnos para lleva

f) Contar con una reglamentación en relación a la modalidad de los exámenes finales excepcionalmente para este período de suspensión de clases presenciales.

g) La necesidad de contar con una planificación para la recup

h) La necesidad de contar con un modelo pedagógico para el desarrollo de las clases virtuales.

i) La revisión del calendario académico de 2021 considerando que se está evaluando en el MEC la modificación del calendario escolar de 2020, lo cual afectaría a egresados del nivel medio, futuros ingresantes en las instituciones de educación superior.

j) Avanzar en el proceso de registro de títulos con gestión online a los efectos de ofrecer un mejor servicio a los usuarios.

Es importante mencionar que en el marco de todas las circunstancias y datos que la realidad fue indicando conforme a la crisis y con el objetivo claro de poder asumir con responsabilidad y con criterio to educación superior, en este periodo, y sobre todo respetando el principio del derecho a la educación el consejo ejecutivo del Consejo Nacional de Educación Superior (CONES) determinó la emisión de la resolución Núm. 08/2020, «que establece pautas generales para las instituciones de educación superior a fin de aplicar herramientas digitales de enseñanza-aprendizaje en el marco de la emergencia

Esta resolución posibilita a las instituciones de educación superior implementar clases a traves de mecanismos tecnológicos para aquellas carreras y materias que estaban siendo impartidas en la modalidad presencial e indica las pautas a seguir, entre las cuales se menciona:

a) Las carreras de pregrado, grado y programas de postgrado deberán estar legalmente habilitadas en la modalidad presencial. 
La situación actual ha motivado a varios estamentos a generar encuentros virtuales para buscar opciones eficaces

y aplicables con las que

responder a los reclamos

y circunstancias que se están

suscitando en la realidad

de la educación superior

b) Las instituciones de educación superior que opten por desarrollar sus ofertas académicas deberán comunicar y remitir al Consejo Nacional de Educación Superior (CONES) la resolución institucional sobre dicha determinación, a fin de que se inserten las carreras de pregrado, grado y programas de postgrado en el registro nacional de ofertas digitales de enseñanza-aprendizaje establecido para el efecto, detallando las carreras y programas que lo han aplicado y las modalidades utilizadas.

c) La aplicación de dichas herramientas digitales de enseñanza-aprendizaje no modificará la calidad o condición de habilitación de las carreras de grado o programas de postgrado como presencial.

d) Los alumnos deberán recibir un servicio educativo de calidad y tener acceso a los recursos de la tecnologia, la información y la comunicación, además de contar con el equipamiento y los materiales adecuados a los requerimientos del aprendizaje mediado, para lo cual la entidad de educación superior debe establecer los mecanismos que acompañen el proceso a través de plataformas digitales de enseñanza y otras herramientas.

e) Establecer un sistema de tutorias -en líneaque desarrolle la interactividad y la atención a las necesidades de aprendizaje de los estudiantes en la utilización de las herramientas digitales de enseñanza-aprendizaje, pudiendo estar disponibles en las páginas digitales de la institución de educación superior.

f) Las instituciones de educación superior deberán contar y remitir al Consejo Nacional de Educación Superior (CONES) los siguientes reglamentos y requisitos a fin de implementar las herramientas digitales de enseñanza-aprendizaje:
1. Resolución institucional que apruebe o disponga la aplicación e implementación de los procesos de enseñanza-aprendizaje en la institución de educación superior.

2. Reglamentación o resolución de evaluación en el marco del proceso de implementación o aplicación de las herramientas digitales de enseñaza-aprendizaje que contemple el proceso de valoración del aprendizaje en forma continua e integra sea este de proceso o de producto. Los registros de evaluación deberán realizarse en forma digital y físca. Será responsabilidad de cada institución garantizar la conservación de dichos registros académcos. Los exámenes o evaluaciones finales deberá ser realizados con las validaciones documentales pertinentes y el proceso deberá quedar registrado en la institución.

3. Reglamentación o resolución que establezca un equipo interdisciplinario de docentes y profesionales para cumplir con los servicios pedagógicos que demanda la implementación del proyecto académico aplicando el sistema digital de enseñanza-aprendizaje.

4. Reglamentación o resolución referente a los procesos de expedición y conservación documental de las actas de exámenes o evaluaciones parciales y finales.

5. Reglamento que establezca las pautas y orientaciones académicas a ser aplicadas para las asignaturas que requieran prácticas, en especial aquellas del área de ciencias de la salud. Dicho reglamento deberá contemplar un análisis y fundamento académico que permita al estudiante la adquisición de las competencias exigidas en la malla curricular respecto de las asignaturas prácticas e incluir los procesos y mecanismos de adecuación o recalendarización ser aplicados.

6. Acreditar la afectación, mediante resolución institucional, de un sistema de soporte tecnológico indicando los responsables de la infraestructura tecnológica y su soporte técnico, asi como de la administración y uso de las plataformas virtuales aplicadas y sus herramientas tecnologicas.

Como podemos ver, en la normativa citada se prevén varias medidas que deben ser desarrolladas por las instituciones de educación superior (IES) a os efectos de garantizar la calidad y la pertinencia de los senvicios académicos prestados, por ejemplo, se debe establecer el mecanismo de evaluación que contemple el proceso de valoración de aprendizaje en forma continua e integral, y elaborar un reglamento que establezca las pautas y orientaciones académicas a ser aplicadas para las asignaturas que requieran prácticas, en especial aquellas del área de ciencias de la salud.

Otra cuestión es la que tiene que ver con los aranceles, sobre todo de las instituciones de edu- cación superior privadas, tal y como también sucede con las instituciones educativas privadas de lo quemás niveles, pues los padres y alumnos exige qualidad de muchas familias cuyos ingresos se ven fectados por la recesión provocada por la cuarentena. Teniendo en cuenta esta situación es que ya varias instituciones de educación superior por me dio de resoluciones de su Consejo Superior Universitario han determinado la reducción de sus aranceles, así como también han propuesto planes de pago a sus estudiantes $y$ descuentos importantes en sus matrículas.

En este sentido, considero importante atender a lo establecido por la Constitución nacional del Paraguay en el artículo 79 sobre las universidades e institutos superiores: «Las universidades son autónomas. Establecerán sus estatutos y formas de gobierno elaborarán sus planes de estudio de acuerdo con la política educativa y los planes de desarrollo nacional. Se garantiza la libertad de enseñanza y la de la cátedra. Las universidades, tanto públicas como privadas, serán creadas por ley, la cual determinará las profesiones que necesiten títulos universitarios para su ejercicio».

Lo que me gustaría señalar con esto es que el Estado no tiene la facultad de regular los aranceles de las universidades bajo ningún instrumento legal observando lo establecido como principio constitucional en la autonomía universitaria.

Como ya mencioné en el libro de mi autoría Entre autonomía y libertad -manual de educación superior-, la autonomía universitaria es esencialmente la libertad de enseñar, investigar y difundir la cultura. sta autonomia académica no existiría de un modo completo si la universidad no tuviera el derecho de organizarse, de funcionar y de aplicar sus recursos conómicos como lo estime más conveniente, es decir, si no poseyera una autonomía administrativa si no disfrutara de una autonomia legislativa, que es su capacidad para dictarse sus propios ordenamientos. Es asi que estos fundamentos deben ser asegurados como garantías constitucionales.

Como ocurre con las instituciones educativas de los demás niveles, la educación superior pública privada, en constante comunicación con el Ministero de Educación y Ciencias, están generando varias euniones para fortalecer dicha educación superior en esta circunstancia de pandemia. Estas reuniones entre los representantes de asociaciones de universidades publicas y privadas, de institutos de educación superior públicos y privados, de rectores de universidades públicas y privadas, como tambien del Consejo de Rectores y de Directores Generaes de Institutos de Educación Superior se realizan sobre temas concretos con la intención de poder desde lo académico, financiero y tecnológico. Es así que se produjeron reuniones virtuales con el Ministerio de Hacienda sobre temas como la posibilidad de direccionamiento de las becas, el descuento o exoneración de tributos, el subsidio para pago de salario a docentes. Desde lo académico, en un trabajo en conjunto entre el Ministerio de Educación y Ciencias y las instituciones de educación superior, se está verificando el desarrollo de las clases con las herramientas tecnológicas y cómo mejorar las experiencias, capacitación a los docentes, conectividad de docentes, estudiantes y todos los usuarios del sistema de educación superior.

\section{Fuentes ybibliografía}

Anelli, Franco (2020): El riesgo de educar «de verdad». Disponible en: https://espanol.clonline.org/noticias/ en-clase/2020/02/11/el-riesgo-de-educar-de-verdad (fecha de consulta: 9 de octubre de 2020).

Carrón, Julián (2020): El despertar de lo humano. Reflexiones de un tiempo vertiginoso. Fraternidad de Comunión y Liberación. Disponible en: https: /lit.clonline. dff(fecha de $20104 / 23 /$ eldespertardelohumano-es, parlect ha de consulta: 9 de octubre de 2020 .

Tonucci: "No perdamos seste tiempo preciosancesco deberes" "(entrevista) en el diario ElPás (España) Disponible en: htps://elpais.com/sociedad/2020-04.11/ dand 2020

Portal del Ministerio de Educación y Ciencias de Paraguay: https://www.mec.gov.py/cms/

coronaviu (2020). «Estudar online en tiempos de coronavirus», en la revista La Verdad (España). Disponible en. heps./Mnnulaverdad.es/sociedad/ educacion/estudiar-online-tiempos-coronavirus-opi 2Fwww.google.com\%2F (fecha de consulta: 9 de junio de 2020).
(2020). COVID-19 y educación superior. Caracas: Instituto Internacional de la UNESCO para la educación superior en América Latina y el Caribe (IESALC).

elázquezSeiferheld, David(2020): «Lagripeespañolallega al Paraguay: 1918", en el diario La Nación. Asunción. Disponible en: https://www.lanacion.com.py/periodicola-gripe-espanola-llega-al-paraguay-1918/ (fecha de consulta: 9 de junio de 2020).

Anexos

Normativas relacionadas al ámbito de educación superior la facultad de las instituciones de educación superior para aplicar herramientas digitales de enseñanza-aprendizaje en el marco de la emergencia 
sanitaria -COVID-19- dispuesta por las autoridades nacionales». Disponible en: www.cones.gov.py/ resolucion-ce-cones-n-042020-consejo-ejecutivoque-establece-la-facultad-de-las-institucionesde-educacion-superior-para-aplicar-herramientasdigitales-de-ensenanza-aprendizaje-en-el/

Resolución CE-CONES Núm. 08/2020 «Que establece pautas generales para las IES en la aplicación de herramientas digitales». Disponible en: http://www.cones.gov.py/resolucion-ce-cones-n-08-que-establecepautas-generales-para-las-ies-en-la-aplicacion-deherramientas-digitales/

\section{Normativas relacionadas a las medidas adoptadas por el Ministerio de Educación y Ciencias}

Resolución 308 de fecha 10 de marzo de 2020 «por la cual se dispone la suspensión de las clases en las instituciones de gestión oficial, privada y privada subvenciona$\mathrm{da}$, de los niveles y modalidades correspondientes a cartera de Estado, por el término de quince (15) días, en el marco de la implementación de acciones preventivas antes el riesgo de expansión del coronavirus (COVID-19), en el territorio nacional». Disponible en: https://www.mec.gov.py/cms v4/documentos/ver documento/?titulo=308-2020-PETTA1

Resolución 306 de fecha 09 de marzo de 2020 «por la cual se aprueba el protocolo de acciones preventivas denominado "Practicamos hábitos saludables para prevenir enfermedades". Asimismo, se dispone su aplicación obligatoria en las instituciones educativas de gestión oficial, privada y privada subvencionada, de todos los niveles y modalidades del sistema educativo nacional». Disponible en: https://www. mec. gov.py/cms v4/documentos/ver documento/?titulo= 306-2020-PETTA1

Resolución 346 de fecha 23 de marzo upor la cual se aprueba el uso de las cuentas y aplicaciones de la plataforma digital Office 365, bajo el dominio del Ministerio de Educación y Ciencias, como instrumento de apoyo para el desarrollo de las clases en las instituciones educativas del país». Disponible en: https://www.mec. gov.py/cms v4/documentos/ver_documento/?titulo= 346-2020-PETTA1

Resolución 349 de fecha 24 de marzo upor la cual se disponen medidas de contingencia a ser aplicadas por las instituciones educativas de gestión privada y privada subvencionada, a los efectos de asegurar el servicio educativo a los estudiantes y el cumplimiento del calendario académico correspondiente, en el marco de la emergencia sanitaria declarada ante el riesgo de expansión del coronavirus (COVID-19), en el territorio nacional». Disponible en: https://www. mec.gov.py/cms v4/documentos/ver documento/? titulo=349-2020-PETTA1
Resolución 351 de fecha 25 de marzo de 2020 «por la cual se autoriza a la dirección general de bienestar estudiantil, dependiente del viceministerio de Educación Básica de esta cartera de Estado, a coordinar la entrega de los kits de alimentos a los estudiantes de las instituciones educativas de gestión oficial y privada subvencionada que reciben alimentación escolar, en el marco de la emergencia sanitaria declarada ante el riesgo de expansión del coronavirus (COVID-19), en el territorio nacional». Disponible en: https://www. mec.gov.py/cms v4/documentos/ver documento/? titulo $=351-2020$-PETTA1

Resolución 354 de fecha 28 de marzo de 2020 «por la cual se extiende el período de suspensión de clases en las instituciones de gestión oficial, privada y privada subvencionada, de los niveles y modalidades correspondientes a esta cartera de Estado, hasta el 12 de marzo de 2020, en el marco de la emergencia sanitaria declarada ante el riesgo de expansión del coronavirus (COVID-19), en el territorio nacional». Disponible en: https://www.mec.gov.py/cms v4/documentos/ ver documento/?titulo=354-2020-PETTA1

Resolución 358 de fecha 27 de marzo de 2020 «por la cual se aprueba la implementación de la plataforma de recursos digitales "Tu escuela en casa" para estudiantes de los niveles de educación inicial, escolar básica y media, como mecanismo de apoyo para el desarrollo de las clases de las instituciones educativas del país». Disponible en: https://www.mec.gov.py/cms v4/ documentos/ver documento/?titulo $=358-2020$ PETTA1

Resolución 372 de fecha 8 de abril de 2020 «por la cual se establece el criterio para la entrega de los kits de alimentos o suplementos nutricionales a los estudiantes de las instituciones educativas de gestión oficial y privada subvencionada que reciben alimentación escolar, en el marco de la emergencia sanitaria declarada ante el riesgo de expansión del coronavirus (COVID-19), en el territorio nacional». Disponible en: https://www.mec.gov.py/cms v4/documentos/ver documento/?titulo=372-2020-PETTA1

Resolución 374 de fecha 8 de abril de 2020 «por la cual se dispone la actualización de los datos de estudiantes matriculados en las instituciones educativas de gestión oficial, privada y privada subvencionada del país, en el registro único del estudiante (RUE)». Disponible en: https://www.mec.gov.py/cms v4/documentos/ver do cumento/?titulo=374-2020-PETTA1

Resolución 423 de fecha 24 de abril «por la cual se integra la mesa técnica de trabajo entre autoridades del Ministerio de Educación y Ciencias y representantes de las organizaciones estudiantiles». Disponible en: https://www.mec.gov.py/cms v4/documentos/ver documento/?titulo=423-2020-PETTA1 